BULL. AUSTRAL. MATH. SOC.

VOL. 9 (1973), 141-143.

\title{
Matchings in graphs
}

\section{P.J. McCarthy}

\begin{abstract}
Results of Tutte and of Anderson giving conditions for a simple graph $G$ to have a perfect matching are generalized to give conditions for $G$ to have a matching of defect $d$. A corollary to one of these results is a theorem of Berge on the size of a maximum matching in $G$.
\end{abstract}

Let $G$ be a simple graph with vertex-set $V$ and edge-set $E$. A matching in $G$ is a set $M$ of edges of $G$ such that every vertex of $G$ is incident to at most one edge in $M$. A matching $M$ in $G$ is perfect if every vertex of $G$ is incident to exactly one edge in $M$.

If $S \subseteq V$, denote by $G_{S}$ the subgraph of $G$ obtained by removing all of the vertices in $S$ and all of the edges to which vertices in $S$ are incident. Denote by $p(S)$ the number of components of $G_{S}$ having an odd number of vertices. In [4] Tutte obtained the following result.

TUTTE'S THEOREM. The graph $G$ has a perfect matching if and only if $p(S) \leq|S|$ for every subset $S$ of $V$.

The simplest proof of this theorem is the recent one by Anderson [1]. It makes use of Hall's Theorem on systems of distinct representatives. Earlier, Berge obtained Tutte's Theorem as a corollary to a result on the number of vertices of $G$ not incident to the edges in a maximum matching in $G$ [2; 3, Chapter 18]. We shall derive this result of Berge from Tutte's Theorem by using a technique popular in transversal theory.

A matching $M$ in $G$ has defect $d$ if there are $d$ vertices of $G$ not incident to edges in $M$.

Received 9 April 1973. The author acknowledges partial support from a University of Kansas General Research Fund grant. 
THEOREM 1. Let $d$ be an integer with $0 \leq d \leq|v|$ and $|v|+d$ even. The groph $G$ has a matching of defect $d$ if and only if $p(s) \leq|s|+d$ for every subset $S$ of $V$.

Proof. Let $D$ be a set such that $|D|=d$ and $V \cap D$ is empty. Let $G^{\prime}$ be the graph with vertex-set $V \cup D$ and edge-set consisting of $E$ and edges joining each element of $D$ with each vertex in $V$. It is clear that $G$ has a matching of defect $d$ if and only if $G^{\prime}$ has a perfect matching. By Tutte's Theorem this is the case if and only if $p^{\prime}(S) \leq|S|$ for every subset $S$ of $V \cup D$, where $p^{\prime}(S)$ is the number of components of $G_{S}^{\prime}$ having an odd number of vertices.

If $V \Xi S$, then $p^{\prime}(S)=|D \backslash S| \leq|D|=d \leq|V| \leq|S|$.

If $V \notin S$ and $D \notin S$, then $p^{\prime}(S)=0$ or. 1 since for $v_{1}, v_{2} \in V \backslash S$ and $x \in D \backslash S$ both $\left\{v_{1}, x\right\}$ and $\left\{v_{2}, x\right\}$ are edges of $G^{\prime}$. If $S$ is empty, then $p^{\prime}(S)=0$ since $|V|+d$ is even. Hence, we always have $p^{\prime}(S) \leq|S|$.

If $D \subseteq S$, let $S=T \cup D$, where $T \subseteq V$. Then $p^{\prime}(S)=p(T)$, and so $G^{\prime}$ has a perfect matching if and only if for all such $S$ we have $p(T)=p^{\prime}(S) \leq|S|=|T|+d$.

A matching $M$ in $G$ is a maximum matching if no matching in $G$ has more edges than $M$.

COROLLARY (Berge). The number of vertices of $G$ not incident to any of the edges in a maximum matching in $G$ is equal to $\max \{p(S)-|S|: S \subseteq V\}$.

Note that if $d$ is the stated maximum, then $|v|+d$ is even: in fact, for every $S \subseteq V$, the quantity $|V|+p(S)-|S|$ is even.

Let $|V|=2 n$. Anderson [1] obtained an interesting sufficient condition for there to exist a perfect matching in $G$, namely, that any $k \leq \frac{3}{2} n$ vertices of $G$ be adjacent to at least $\frac{4}{3} k$ vertices. We offer the following generalization.

THEOREM 2. Let $d$ be an integer such that $0 \leq d \leq \frac{3}{4}|V|$ and $|V|+d$ is even. If any $k \leq \frac{3}{4}(|V|+d)$ vertices of $G$ are adjacent to at least $\frac{4}{3} k-d$ vertices, then $G$ has a matching of defect $d$. 
Proof. Let $d$ and $G^{\prime}$ be as in the proof of Theorem 1 . By Anderson's result, $G^{\prime}$ has a perfect matching if any $k \leq \frac{3}{4}(|v|+d)$ vertices of $G^{\prime}$ are adjacent to at least $\frac{4}{3} k$ vertices of $G^{\prime}$. We show that this is the case when $G$ satisfies the hypothesis of the theorem. Let $S \subseteq V \cup D$ be such that $|S|=k \leq \frac{3}{4}(|V|+d)$.

If $S \subseteq D$, then the vertices in $S$ are adjacent to the $|V|$ vertices in $V$, and $k \leq d \leq \frac{3}{4}|V|$ : hence $\frac{4}{3} k \leq|V|$.

If $S \notin V$ and $S \notin D$, then the vertices in $S$ are adjacent to the $|V|+d$ vertices of $G^{\prime}$, and $\frac{4}{3} k \leq|V|+d$.

If $S \subseteq V$, then the vertices in $S$ are adjacent to the $d$ vertices of $G$ in $D$ and to at least $\frac{4}{3} k-d$ vertices in $V$. Hence, the vertices in $S$ are adjacent to at least $\frac{4}{3} k$ vertices of $G^{\prime}$.

As a corollary we obtain a result for graphs with an odd number of vertices analogous to Anderson's result for graphs with an even number of vertices.

COROLLARY. If $|V|=2 n+1$, and if any $k \leq \frac{3}{2}(n+1)$ vertices of $G$ are adjacent to at least $\frac{4}{3} k-1$ vertices, then $G$ has a matching of defect one.

\section{References}

[1] Ian Anderson, "Perfect matchings of a graph", J. Combinatorial Theory Ser. B 10 (1971), 183-186.

[2] Claude Berge, "Sur le couplage maximum d'un graphe", C.R. Acad. Sci. Paris 247 (1958), 258-259.

[3] Claude Berge, The theory of graphs and its applications (translated by Alison Doig. Methuen,. London; John Wiley \& Sons, New York, 1962).

[4] W.T. Tutte, "The factorization of linear graphs", J. London Math. Soc. 22 (1947), 107-111.

Department of Mathematics, University of Kansas, Lawrence, Kansas, USA. 